\title{
Treatment options in end-stage heart failure: where to go from here?
}

\author{
M. L. A. Haeck • G. E. Hoogslag • S. F. Rodrigo • \\ D. E. Atsma • R. J. Klautz • E. E. van der Wall • \\ M. J. Schalij • H. F. Verwey
}

Published online: 15 November 2011

(C) The Author(s) 2011. This article is published with open access at Springerlink.com

\begin{abstract}
Chronic heart failure is a major healthcare problem associated with high morbidity and mortality. Despite significant progress in treatment strategies, the prognosis of heart failure patients remains poor. The golden standard treatment for heart failure is heart transplantation after failure of medical therapy, surgery and/or cardiac resynchronisation therapy. In order to improve patients' outcome and quality of life, new emerging treatment modalities are currently being investigated, including mechanical cardiac support devices, of which the left ventricular assist device is the most promising treatment option. Structured care for heart failure patients according to the most recent international heart failure guidelines may further contribute to optimal decision-making. This article will review the conventional and novel treatment modalities of heart failure.
\end{abstract}

Marlieke L.A. Haeck and Georgette E. Hoogslag both contributed equally to this review and share first authorship

Electronic supplementary material The online version of this article (doi:10.1007/s12471-011-0211-4) contains supplementary material, which is available to authorized users.

M. L. A. Haeck · G. E. Hoogslag • S. F. Rodrigo • D. E. Atsma •

E. E. van der Wall $\cdot$ M. J. Schalij $(\bowtie) \cdot H$. F. Verwey

Department of Cardiology, Leiden University Medical Center,

Albinusdreef 2,

2333 ZA Leiden, the Netherlands

e-mail: m.j.schalij@lumc.nl

R. J. Klautz

Department of Cardiothoracic Surgery,

Leiden University Medical Center,

Albinusdreef 2,

2333 ZA Leiden, the Netherlands
Keywords Heart failure $\cdot$ Surgery $\cdot$ Resynchronization therapy $\cdot$ Mechanical cardiac support $\cdot$ Heart failure program

\section{Introduction}

Chronic heart failure (HF) is a major healthcare problem associated with high morbidity and mortality. In the Netherlands, an estimated 180,000 patients in a population of 16.5 million suffer from HF with an incidence rate of 35,000 patients per year [1]. Over the last two decades, no change has been observed in the incidence of HF, despite better prevention and treatment options [2]. Notwithstanding significant progress in treatment modalities, the prognosis of HF patients still remains poor. It was demonstrated that patients with drug refractory end-stage HF receiving support with inotropic drugs had a 3-, 6-, and 12-month survival of $51 \%, 26 \%$, and $6 \%$, respectively, with a mean survival of only 3.4 months [3]. For these patients, heart transplantation (HTX) remains the only option $[4,5]$. In comparison with conventional treatment, HTX significantly provides a better quality of life and increases survival: it is associated with a $90 \%$ 1-year and a $80 \%$ 3-year survival [6].

Nevertheless, only a fraction of the HTX candidates will actually benefit from transplantation, as the number of available donor hearts is limited. Moreover, strict selection criteria result in the exclusion of many candidates.

However, new surgical techniques, cardiac resynchronisation therapy (CRT), and mechanical cardiac support may emerge as promising options for this patient group. Because of the large number of treatment options, it is important that before treatment, HF patients should always enter a 
structured screening protocol, embedded in a specialised HF program, to determine the aetiology of HF and to identify possible treatment options.

\section{Pharmacological therapy and lifestyle changes}

The aim of HF treatment is to prevent progression of symptoms and to improve survival. Treatment of HF is based on two cornerstones: lifestyle intervention and pharmacological therapy. Lifestyle changes include restriction of sodium and fluid intake, losing weight, cessation of smoking and alcohol, and participation in a rehabilitation program [4]. Besides optimal control of risk factors (e.g. hypertension, coronary artery disease, and diabetes mellitus), drug treatment of HF includes angiotensin-converting enzyme (ACE) inhibitors or, when not tolerated, angiotensin II receptor blockers. Furthermore, diuretics, aldosterone receptor antagonists, digoxin, and beta-blockers are important in the pharmacological treatment of HF. In the past decades, several large clinical randomised trials have demonstrated the value of these drugs in reduction of mortality, improvement of functional status, and reduction of the number of hospitalisations [4].

\section{Surgical treatment}

In addition to pharmacological therapy, novel surgical techniques to alleviate HF have been introduced. These surgical techniques address different pathophysiological mechanisms in HF. Revascularisation may potentially improve the outcome of patients with ischaemic cardiomyopathy. Despite the significant periprocedural risk of morbidity and mortality, several studies demonstrated a higher survival rate and improved New York Heart Association (NYHA) functional class in patients with HF after coronary artery bypass grafting (CABG) compared with patients on optimal medical therapy only $[7,8]$.

In patients with ischaemic cardiomyopathy, LV remodelling, characterised by an increase in LV volume and a deteriorating cardiac function, is associated with progression of HF and poor prognosis [9]. Surgical ventricular restoration (SVR) aims to correct ventricular remodelling by restoring LV geometry and reducing its volume, thus improving LV function (Fig. 1) [10, 11]. The RESTORE study examined early and late outcome after SVR in 1,198 postinfarction patients. In this study, there was an improvement of approximately $10 \%$ in LV ejection fraction and all patients had a decrease in symptoms. After surgery, 30-day mortality was $5.3 \%$ and overall 5 -year survival was $68.6 \%$ [12]. In the STICH trial, 1,000 patients with an LV ejection fraction $<35 \%$ were randomly assigned to $\mathrm{CABG}$ in combination with SVR or CABG alone [13]. Primary endpoints were death and hospitalisation for cardiac causes. A significant reduction $(19 \%)$ of the end-systolic volume index was found in the SVR group compared with a $6 \%$ reduction in the $\mathrm{CABG}$ group. However, no difference in reduction of cardiac symptoms or improvement of exercise tolerance was observed. Furthermore, SVR did not reduce the rate of death or hospitalisation for cardiac causes compared with $\mathrm{CABG}$ alone.

Another surgical intervention that addresses ventricular remodelling is the implantation of a CorCap Cardiac Support Device (CSD; Acorn Cardiovascular Inc, St. Paul, MN, US). The CorCap CSD is a passive external fabric mesh containment device that is surgically implanted around the heart in order to reduce LV wall stress by providing circumferential diastolic support. In the Acorn trial, the safety and efficacy of the CorCap CSD was assessed in 300 NYHA class III-IV HF patients. Patients were excluded if there was a need for other cardiac surgical procedures than mitral valve replacement. The use of the CorCap CSD resulted in a significant decrease of LV volumes and LV sphericity and improved functional status and quality of life [14]. During 3 years of follow-up, these changes were maintained, demonstrating the long-term beneficial effect of the CorCap CSD on reverse remodelling in HF patients [15].

Functional mitral regurgitation (MR) frequently develops in patients with chronic HF. In 2,057 HF patients from the Duke Cardiovascular Databank, MR of any grade was present in almost $60 \%$ [16]. In HF patients, functional MR develops because of LV dilatation and deformation, which leads to incomplete leaflet coaptation. The development of MR is associated with worsening of symptoms and decreased survival [16]. Conservative management of severe to moderate functional MR was associated with a 5 -year survival of approximately $40 \%$, independent of the aetiology, compared with a 5 -year survival rate of $55 \%$ in HF patients without MR [16]. Mitral valve surgery aims to treat MR by undersizing the mitral annulus by a restrictive mitral annuloplasty (RMA). In a study of 51 patients with ischaemic LV dysfunction and severe MR, combined RMA and CABG led to reduced LV remodelling and symptoms and a 2-year survival of $84 \%$ [17]. However, despite these promising data, no large randomised controlled trials have been performed to address the long-term outcome of this procedure as compared with other therapeutic options.

\section{Cardiac resynchronisation therapy (CRT)}

An important development has been the implementation of CRT. In approximately $25 \%$ of patients with HF, intraventricular conduction delay is apparent [18], resulting in 


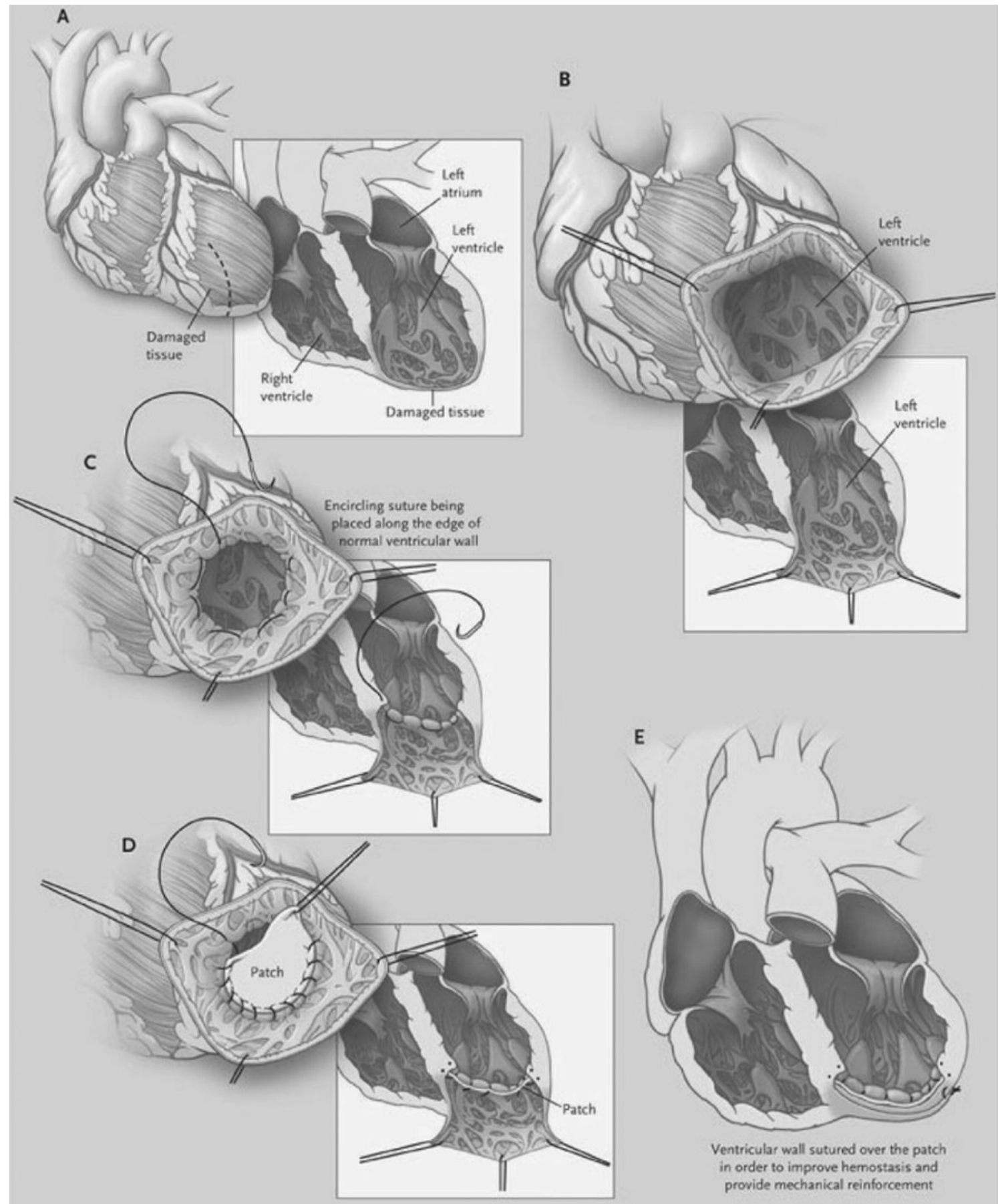

Fig. 1 Surgical restoration of the left ventricle which aims to restore ventricular remodelling by reshaping left ventricular geometry and reducing its volume. This results in an improvement in the function of

asynchronous ventricular contraction. Asynchrony may further reduce systolic function and cardiac output in the already challenged heart [19]. In this light, CRT (first introduced by Dr. Bakker from Utrecht, the Netherlands) has emerged as a well-established therapy for patients with advanced HF. Cardiac stimulation resynchronises ventricular contraction thereby improving LV systolic function. the left ventricle. Figures A to E demonstrate the consecutive steps of this procedure (Reprinted with permission of Eisen) [11]

Current indications for CRT are NYHA functional class III to IV, LV ejection fraction $<35 \%$, and a QRS duration $>120 \mathrm{~ms}$ [4]. Two large randomised clinical trials, MADIT-CRT [20] and REVERSE [21], evaluated CRT in mild to moderate HF patients and demonstrated significant reverse remodelling and a significant reduction in HF events. Based on this, the guidelines also recommended CRT in patients with NYHA 
functional class II [22]. A positive effect of CRT was demonstrated on symptoms of HF, functional capacity and quality of life [23]. Furthermore, CRT significantly reduces mortality and hospitalisation rate due to HF [24]. However, one third of the patient population with CRT does not show response in terms of reverse LV remodelling and clinical improvement [25].

Despite the current established treatment options, including drugs, surgery and CRT, a significant number of patients will experience deteriorating symptoms and eventually die. Therefore, it is hopeful that mechanical cardiac support, a promising innovative treatment option, may improve the outcome of these patients.

\section{Mechanical cardiac support}

In 1966, the first ventricular assist device (VAD) was implanted by DeBakey as bridge to recovery. This was the beginning of mechanical circulatory support for advanced HF.

VADs are mechanical circulatory pumps which partially or completely take over ventricular function in order to assist the circulation and improve end-organ perfusion. A VAD can be used as an LV (LVAD), right ventricular (RVAD), and as biventricular assist device (BiVAD). Mechanical circulatory support is used for three indications [26]. (1) As bridge to HTX, in which a patient receives a VAD for a relatively short time in order to survive the waiting list period until transplantation and to prevent further deterioration and even improve secondary organ function. (2) As bridge to recovery, in which the device is implanted in order to allow the heart to recover in, for example, patients with post-cardiotomy shock or peripartum cardiomyopathy. Patients are expected to fully recover and mechanical circulatory support is usually short term. (3) As destination therapy, when recovery cannot be expected and the patient is not a candidate for HTX. Especially with the new-generation of devices, VAD therapy may become an important treatment modality on top of optimal medical therapy, surgery and/or CRT.

The first generation of assist devices were large paracorporal pulsatile devices. These pumps could be used in both ventricles. The large pump chamber was placed on the abdominal wall of the patient and connected by conduits to the great arteries. Besides numerous complications due to the size of the devices, patients were bedridden [26, 27]. Miniaturisation of the control and power-supply components and technological advances resulted in smaller wearable pulsatile VADs that were implanted intra-abdominally. These pumps were either pneumatically or electrically driven [26, 27]. Because of their smaller implantable size, patients could mobilise, rehabilitate and even leave the hospital [28]. However, because of the large volume of the pump only patients with a body surface area $>1.5 \mathrm{~m}^{2}$ were able to receive these mechanical assist devices. Moreover, extensive surgery was necessary because of the size, increasing the risk of haematomas and infection [29]. Due to multiple moving parts the devices were prone to fail and could only be used for a limited amount of time. Anticoagulation was required except for the HeartMate XVE.

The second-generation assist devices consist of smaller axial continuous flow pumps (Fig. 2). Therefore, they require less extensive surgery reducing the risk of perioperative complications. Because these devices contain only one moving part they are more durable than pulsatile devices and noiseless $[27,30]$. During development of the continuous flow pump, there was concern that continuous flow and therefore continuous end-organ perfusion could not be tolerated by the body. This has been contradicted by demonstrating superior survival and less organ failure in patients with continuous flow VADs compared with patients with pulsatile VADs [30].

Further development led to the third-generation devices consisting of even smaller centrifugal blood pumps (Fig. 3a and $b$ ). These pumps have magnetically levitated rotors preventing mechanical wear (Fig. 4). Therefore, it is expected that durability is significantly improved compared with older devices. Because of the small size, the pumps can be implanted intrapericardially further reducing postoperative complications. The first report on the clinical use of the HeartWare ventricular assist device (HVAD) pump (HeartWare Inc, Framingham, MA), a third-generation device, showed in 23 patients promising results with a 6-month and 1 -year survival of $91 \%$ and $86 \%$, respectively [31]. Infection and bleeding were found to be the most common adverse events. Another multicentre trial using a third-generation VentrAssist LVAD (Ventracor Ltd, Chatswood, NSW, Australia) in 33 patients demonstrated a favourable efficacy and safety profile for the use of this device as bridge to transplant [32]. After a median support time of 142 days, $82 \%$ of the patients were either transplanted or remained eligible for transplant. Major adverse events were infections, nonneurological thromboembolic events, neurological events, and haemorrhages. There was a significant improvement in quality of life and $81 \%$ of the patients achieved NYHA class I or II at the end of the trial [32].

\section{Bridge to HTX}

Since the introduction of LVAD therapy as bridge to transplant in the 1980s, this concept has been evaluated in several studies. One multicentre clinical trial reported a $65 \%$ survival until transplantation of 34 patients who 

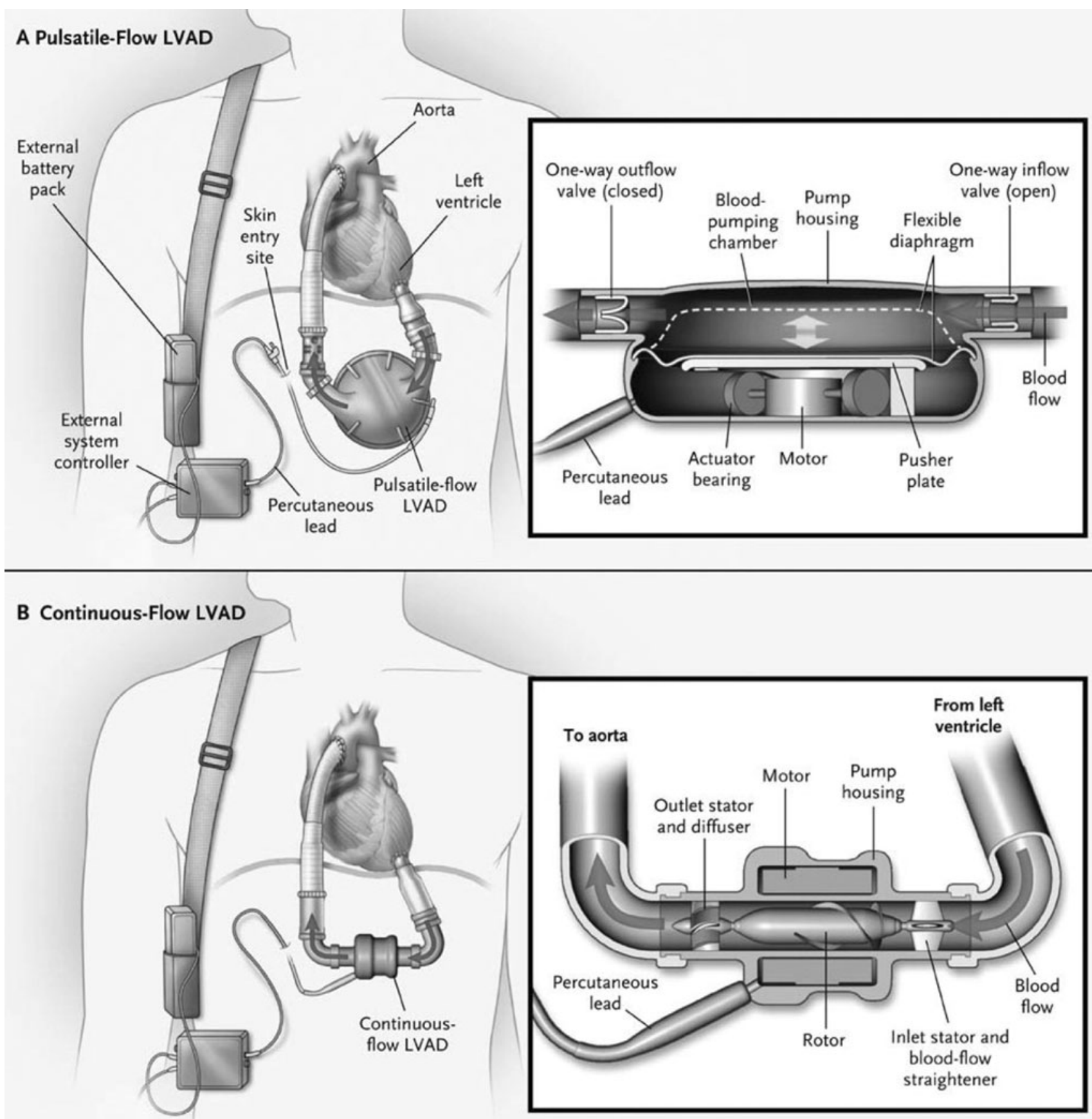

Fig. 2 Panel A shows a first-generation pulsatile flow left ventricular assist device (LVAD). Panel B shows a second-generation continuous flow LVAD. Both mechanical pumps are placed in the abdominal wall. The inflow cannula is placed in the apex of the left ventricle. The

received a LVAD as compared with $50 \%$ in 6 patients who were treated with medication only [33]. Similar results were reported with survival until HTX of $60-75 \%$ [34]. All studies were performed with first-generation pulsatile devices. Although it was demonstrated that haemodynamics and quality of life improved [34], a high adverse event rate was frequently reported. outflow cannula is anastamosed with the ascending aorta. A percutaneous lead connects the LVAD pump with an external system controller and the battery pack (Reprinted with permission of Slaughter et al.) [30]

With the development of the continuous flow assist devices, some of the constraints of the pulsatile devices improved, such as the high device failure rate and associated morbidity. In a multicentre study, 133 NYHA class IV patients received a HeartMate II device as bridge to transplant [35]. Overall survival was $75 \%$ at 6 months and $68 \%$ at 12 months. In this study, major adverse events 


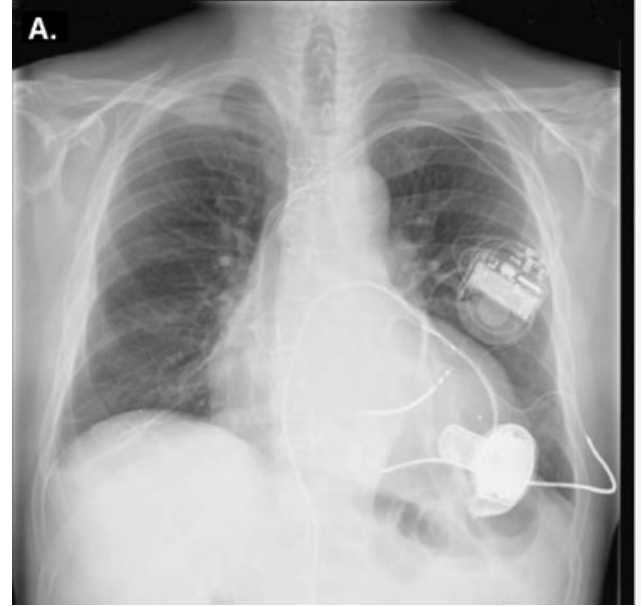

Fig. 3 Third-generation HeartWare ventricular assist device (HVAD). This device consists of a magnetically levitated rotor pump that prevents mechanical wear. The pump is implanted in the pericardial space in the apex of the left ventricle. The outflow graft is connected with the ascending aorta. The controller and external batteries are

were postoperative bleeding, stroke, right HF, and percutaneous lead infection. Five devices were replaced due to pump thrombosis or complications related to surgical implantation.

\section{Bridge to recovery}

In a small number of patients, it has been reported that an LVAD could be explanted because of recovery of myocardial function, allowing the patient to be removed from the waiting list for HTX as well [36]. The general idea of cardiac recovery after LVAD implantation is that unloading of the ventricle leads to reverse remodelling on genetic, molecular and functional levels [37]. The reported frequency varies from $5 \%$ to $29 \%[37,38]$. Recovery of myocardial function seems to be more likely in patients with myocarditis or non-ischaemic cardiomyopathy, e.g. peripartum cardio-

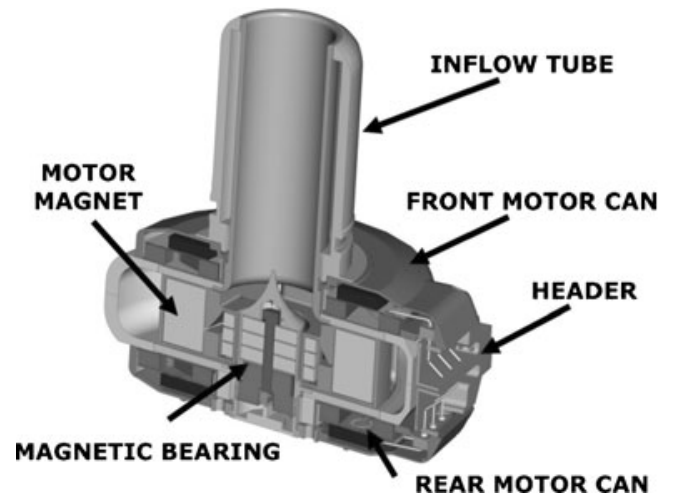

Fig. 4 HeartWare left ventricular assist device pump. The pump has one moving part, the impeller. There are no mechanical bearings (Reproduced with permission of HeartWare Inc.)

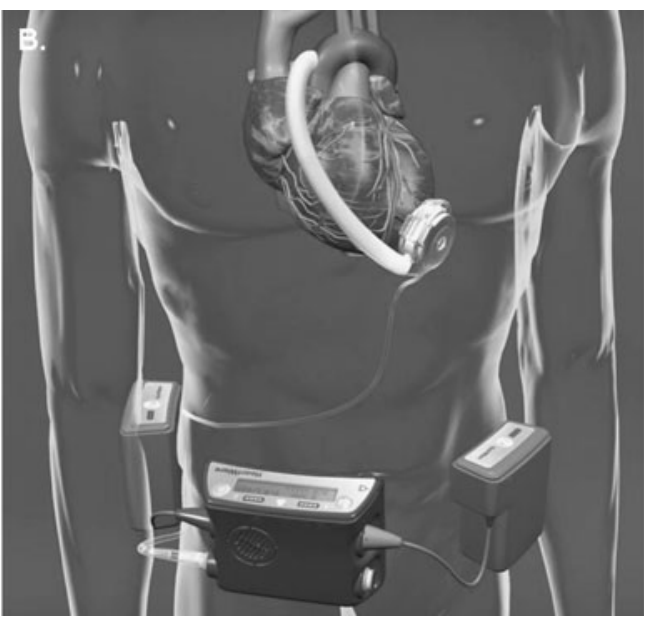

connected to the pump by a percutaneous driveline. In panel A, a chest $\mathrm{x}$-ray is shown of a patient with a HVAD implant and an internal cardioverter defibrillator. Panel B shows the position of all the components of the HVAD system (Panel B reproduced with permission of HeartWare Inc.)

myopathy [38, 39]. However, rapid LV dysfunction after removal of the assist device has been reported [37]. There are no reliable parameters to predict outcome after explantation, nor is there at present a way to predict, before implantation, in which patients cardiac function will recover.

\section{Destination therapy}

Currently, LVADs are implanted as bridge to transplant in most patients. However, since the waiting time on the list for HTX only increases in most Western countries, duration of mechanical support becomes longer. Moreover, some patients who received a LVAD as bridge to transplant removed themselves from the waiting list and continued being on mechanical cardiac support. Therefore, the question is legitimate if it is feasible to use LVADs as destination therapy [40].

In the Randomized Evaluation of Mechanical Assistance for the Treatment of Congestive HF (REMATCH) trial, the feasibility of LVAD therapy as destination therapy was evaluated in 129 patients with end-stage HF who were randomly assigned to receive either optimal medical treatment or LVAD therapy on top of optimal medical therapy [41]. HTX was contraindicated in all patients. A $48 \%$ risk reduction of death from any cause was demonstrated in the LVAD group. Additionally, functional capacity and quality of life were significantly better in the device group compared with the group on optimal medical therapy only. Despite these positive results, serious complications occurred 2.35 times more often in the device group than in the medically treated group. The second study evaluating VADs in non-transplantable patients was the Investigation of Nontransplant-Eligible Patients Who 
Are Inotrope Dependent (INTrEPID) trial [42]. This nonrandomised trial reinforced the results of the REMATCH trial by demonstrating significantly better survival and improvement of symptoms and quality of live in patients with a VAD compared with patients on optimal medical treatment only. However, this study also reported that LVAD implantation resulted in an increased risk of neurological events, infections, and bleeding complications as compared with a medically treated control group of patients [42]. The first randomised trial comparing firstgeneration pulsatile VADs (HeartMate XVE) with secondgeneration continuous flow devices (HeartMate II) in a 1:2 ratio including 200 patients as destination therapy was recently published [30]. The 2-year survival free from disabling stroke, and reoperation to repair or replace LVAD for the continuous group was $46 \%$ compared with $11 \%$ for the pulsatile flow group. Reoperation for pump replacement of the continuous flow LVAD occurred at a rate of 6 events per 100 patient-years, mostly because of damage to the percutaneous lead. No difference in the occurrence of stroke between the two devices was observed. The 1-year actuarial survival rates were $68 \%$ and $55 \%$ for the continuous flow and pulsatile flow group, respectively, and the 2-year survival rates $58 \%$ and $24 \%$, respectively. Both devices improved NYHA functional class and quality of life as compared with baseline characteristics. The continuous flow assist device was associated with a significant reduction in adverse events as compared with the pulsatile assist device. The HeartMate II received Food and Drug Administration approval as destination therapy in 2010.

Since the first implant in 1966, mechanical cardiac support has undergone continuous development. With each new adjustment, the constraints of VADs become less. The longevity of the new devices is expected to be superior to the older devices. Still, there is concern about the risk of adverse events, including bleeding complications and thromboembolic events. More experience in management of these devices may result in fewer complications and better survival.

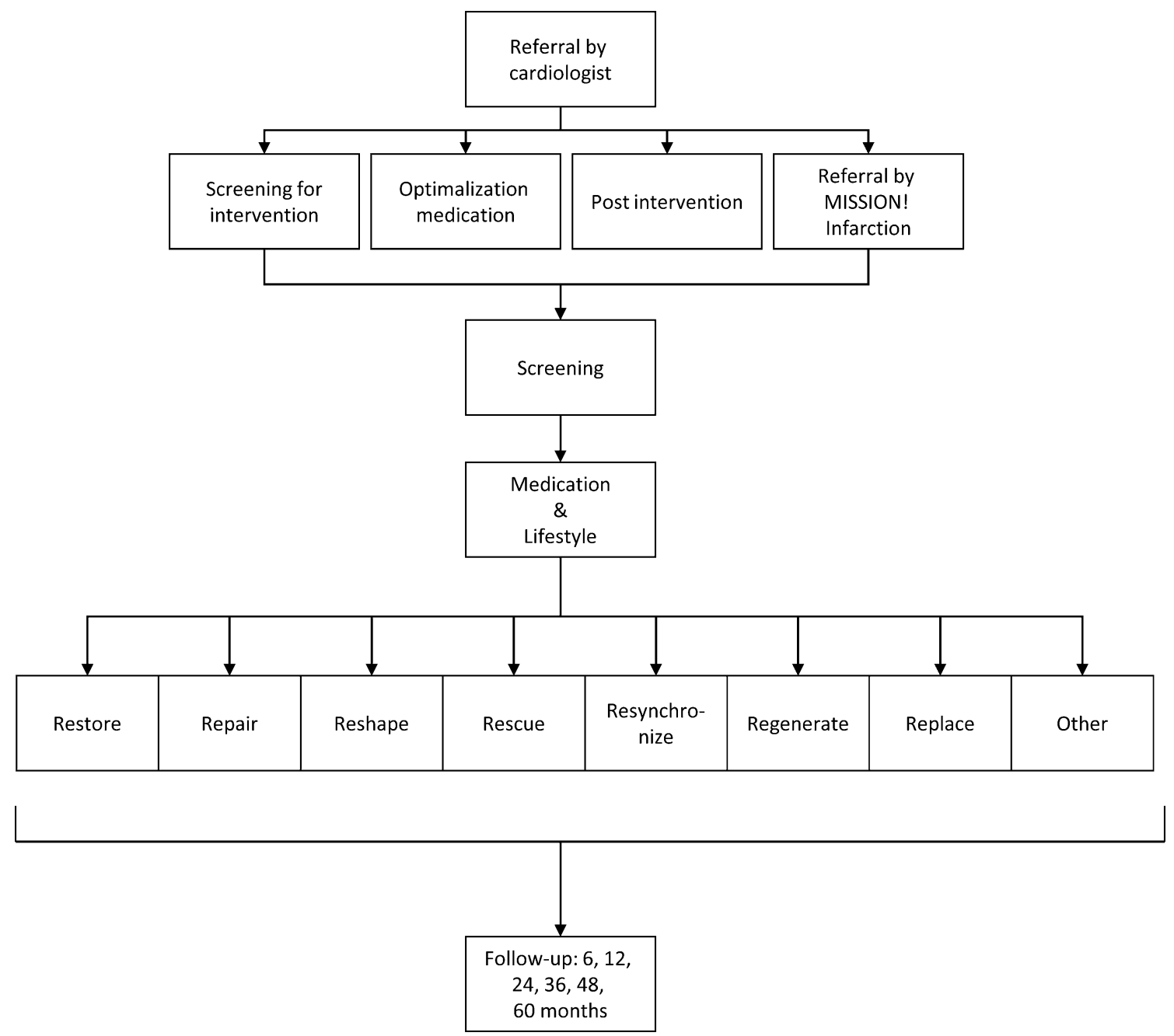

Fig. 5 Comprised flow chart of the MISSION! Heart failure protocol at the Leiden University Medical Centre 


\section{Structured care for patients with HF: MISSION! HF}

$\mathrm{HF}$ is a complex syndrome with a large variety of aetiologies and treatment options, which ranges from pharmaceutical treatment to mechanical support. Not every patient is eligible for all treatment modalities and patients who receive treatment do not always benefit or respond in the desired manner. Therefore, structured screening and treatment procedures embedded within a dedicated and comprehensive HF program are mandatory.

The Leiden MISSION! HF program, initiated in 2005, is an example of such a program (Supplementary Fig. 1). This protocol aims to assess and treat patients according to the most recent guidelines of the European Society of Cardiology for HF [4, 22] and allows implementation of new treatment modalities in order to improve patient care and survival. MISSION! HF is a multidisciplinary approach program with participation of all the care professionals involved.

To achieve an efficient and accurate treatment strategy per patient, patients referred to the MISSION! HF outpatient clinic are extensively screened. This screening program consists of clinical and functional evaluations, including two- and three-dimensional echocardiography, nuclear imaging, MIBG scanning, coronary angiography, and 24-hour ambulatory ECG Holter monitoring.

Guided by the results of the screening program, patients receive tailored treatment according to current guidelines [4, 22]. The treatment includes focus on lifestyle changes, optimal medical treatment and rehabilitation and is led by a specialised HF nurse. Furthermore, in every patient, it is important to question the possibility of revascularisation, valve repair, SVR or implantation of a CorCap. Also, the risk of sudden death is evaluated. Patients who are not eligible for conventional treatment are considered candidates for more advanced treatment options, such as HTX, cell therapy or LVAD support (Fig. 5 and Supplementary Fig. 1).

After screening and start of treatment, patients are followed at the outpatient clinic on a regular basis. During these visits, functional status and treatment is evaluated in a structured manner.

\section{Conclusion}

Progressive symptoms of HF are devastating for both patients and partners. Currently, after failure of medical therapy, surgery and/or CRT, HTX is still the gold standard treatment modality. New treatment options, including mechanical support devices, are under investigation. In patients with end-stage HF, LVAD therapy is currently the most promising treatment option and with the miniaturisation of the devices, it can be expected that the number of implants will rise significantly. In this context, it remains indispensible to individualise patients' treatment strategies.

Acknowledgements The Department of Cardiology received unrestricted grants from Boston Scientific, Medtronic and Biotronik.

Open Access This article is distributed under the terms of the Creative Commons Attribution Noncommercial License which permits any noncommercial use, distribution, and reproduction in any medium, provided the original author(s) and source are credited.

\section{References}

1. Vaartjes I, Reitsma JB, de Bruin A, et al. Verschillen tussen mannen en vrouwen in sterftekans na een eerste ziekenhuisopname vanwege hartfalen. Nederlandse Hartstichting; 2008.

2. Levy D, Kenchaiah S, Larson MG, et al. Long-term trends in the incidence of and survival with heart failure. N Engl J Med. 2002;347(18):1397-402.

3. Hershberger RE, Nauman D, Walker TL, et al. Care processes and clinical outcomes of continuous outpatient support with inotropes (COSI) in patients with refractory endstage heart failure. J Card Fail. 2003;9(3):180-7.

4. Dickstein K, Cohen-Solal A, Filippatos G, et al. ESC Guidelines for the diagnosis and treatment of acute and chronic heart failure 2008. Eur Heart J. 2008;29(19):2388-442.

5. de Jonge N, Kirkels JH, Klopping C, et al. Guidelines for heart transplantation. Neth Heart J. 2008;16(3):79-87.

6. Copeland JG. Advanced medical therapy does not render heart transplantation obsolete for ambulatory end-stage heart failure patients: a debate. J Heart Lung Transplant. 2001;20(7):725-8.

7. Baker DW, Jones R, Hodges J, et al. Management of heart failure: III. The role of revascularization in the treatment of patients with moderate or severe left ventricular systolic dysfunction. JAMA. 1994;272(19):1528-34.

8. Velazquez EJ, Lee KL, Deja MA, et al. Coronary-artery bypass surgery in patients with left ventricular dysfunction. N Engl J Med. 2011;364(17):1607-16.

9. Gaudron P, Eilles C, Kugler I, et al. Progressive left ventricular dysfunction and remodeling after myocardial infarction. Potential mechanisms and early predictors. Circulation. 1993;87(3):755-63.

10. Di Donato M, Sabatier M, Dor V, et al. Effects of the Dor procedure on left ventricular dimension and shape and geometric correlates of mitral regurgitation one year after surgery. J Thorac Cardiovasc Surg. 2001;121(1):91-6.

11. Eisen HJ. Surgical ventricular reconstruction for heart failure. N Engl J Med. 2009;360(17):1781-4.

12. Athanasuleas CL, Buckberg GD, Stanley AWH, et al. Surgical ventricular restoration in the treatment of congestive heart failure due to post-infarction ventricular dilation. J Am Coll Cardiol. 2004;44(7):1439-45.

13. Jones RH, Velazquez EJ, Michler RE, et al. Coronary bypass surgery with or without surgical ventricular reconstruction. N Engl J Med. 2009;360(17):1705-17.

14. Mann DL, Acker MA, Jessup M, et al. Clinical evaluation of the CorCap Cardiac Support Device in patients with dilated cardiomyopathy. Ann Thorac Surg. 2007;84(4):1226-35.

15. Starling RC, Jessup M, Oh JK, et al. Sustained benefits of the CorCap Cardiac Support Device on left ventricular remodeling: three year follow-up results from the Acorn Clinical Trial. Ann Thorac Surg. 2007;84(4):1236-42. 
16. Trichon BH, Felker GM, Shaw LK, et al. Relation of frequency and severity of mitral regurgitation to survival among patients with left ventricular systolic dysfunction and heart failure. Am J Cardiol. 2003;91(5):538-43.

17. Bax JJ, Braun J, Somer ST, et al. Restrictive annuloplasty and coronary revascularization in ischemic mitral regurgitation results in reverse left ventricular remodeling. Circulation. 2004;110 (11_suppl_1):II-103.

18. Baldasseroni S, Opasich C, Gorini M, et al. Left bundle-branch block is associated with increased 1-year sudden and total mortality rate in 5517 outpatients with congestive heart failure: A report from the Italian network on congestive heart failure. Am Heart J. 2002;143(3):398-405.

19. Littmann L, Symanski JD. Hemodynamic implications of left bundle branch block. J Electrocardiol. 2000;33(Supplement 1):115-21.

20. Moss AJ, Hall WJ, Cannom DS, et al. Cardiac-resynchronization therapy for the prevention of heart-failure events. N Engl J Med. 2009;361(14):1329-38.

21. Linde C, Abraham WT, Gold MR, et al. Randomized trial of cardiac resynchronization in mildly symptomatic heart failure patients and in asymptomatic patients with left ventricular dysfunction and previous heart failure symptoms. J Am Coll Cardiol. 2008;52(23):1834-43.

22. Dickstein K, Vardas PE, Auricchio A, et al. 2010 Focused Update of ESC Guidelines on device therapy in heart failure. Eur J Heart Fail. 2010;12(11):1143-53.

23. Young JB, Abraham WT, Smith AL, et al. Combined cardiac resynchronization and implantable cardioversion defibrillation in advanced chronic heart failure: The MIRACLE ICD Trial. JAMA. 2003;289(20):2685-94.

24. Lindenfeld J, Feldman AM, Saxon L, et al. Effects of cardiac resynchronization therapy with or without a defibrillator on survival and hospitalizations in patients with New York Heart Association class IV heart failure. Circulation. 2007;115(2):204-12.

25. Ypenburg C, van Bommel RJ, Borleffs CJW, et al. Long-term prognosis after cardiac resynchronization therapy is related to the extent of left ventricular reverse remodeling at midterm follow-up. J Am Coll Cardiol. 2009;53(6):483-90.

26. Goldstein DJ, Oz MC, Rose EA. Implantable left ventricular assist devices. N Engl J Med. 1998;339(21):1522-33.

27. Christiansen S, Klocke A, Autschbach R. Past, present, and future of long-term mechanical cardiac support in adults. J Card Surg. 2008;23(6):664-76.

28. Oosterom A, de Jonge N, Kirkels JH, et al. End-stage heart failure and mechanical circulatory support: feasibility of discharge from hospital. Neth Heart J. 2007;15(2):45-50.
29. Oz MC, Goldstein DJ, Rose EA. Preperitoneal placement of Ventricular Assist Devices: an illustrated stepwise approach. J Card Surg. 1995;10(4):288-94.

30. Slaughter MS, Rogers JG, Milano CA, et al. Advanced heart failure treated with continuous-flow Left Ventricular Assist Device. N Engl J Med. 2009;361(23):2241-51.

31. Wieselthaler GM, O'Driscoll G, Jansz P, et al. Initial clinical experience with a novel left ventricular assist device with a magnetically levitated rotor in a multi-institutional trial. J Heart Lung Transplant. 2010;29(11):1218-25.

32. Esmore D, Kaye D, Spratt P, et al. A prospective, multicenter trial of the VentrAssist Left Ventricular Assist Device for bridge to transplant: safety and efficacy. J Heart Lung Transplant. 2008;27 (6):579-88

33. Frazier OH, Rose EA, Macmanus Q, et al. Multicenter clinical evaluation of the HeartMate $1000 \mathrm{IP}$ left ventricular assist device. Ann Thorac Surg. 1992;53(6):1080-90.

34. Pennington DG, McBride LR, Peigh PS, et al. Eight years' experience with bridging to cardiac transplantation. J Thorac Cardiovasc Surg. 1994;107(2):472-81.

35. Miller LW, Pagani FD, Russell SD, et al. Use of a continuous-flow device in patients awaiting heart transplantation. N Engl J Med. 2007;357(9):885-96.

36. Frazier MD, Benedict MD, Radovancevic MD, et al. Improved left ventricular function after chronic left ventricular unloading. Ann Thorac Surg. 1996;62(3):675-82.

37. Mancini DM, Beniaminovitz A, Levin $\mathrm{H}$, et al. Low incidence of myocardial recovery after Left Ventricular Assist Device implantation in patients with chronic heart failure. Circulation. 1998;98 (22):2383-9.

38. Simon MA, Kormos RL, Murali S, et al. Myocardial recovery using Ventricular Assist Devices: prevalence, clinical characteristics, and outcomes. Circulation. 2005;112(9 suppl):I-32.

39. Oosterom L, de Jonge N, Kirkels J, et al. Left ventricular assist device as a bridge to recovery in a young woman admitted with peripartum cardiomyopathy. Neth Heart J. 2008;16(12):426-8.

40. Pruijsten RV, de Jonge N, Kirkels JH, et al. Left ventricular assist device: a functional comparison with heart transplantation. Neth Heart J. 2008;16(2):41-6.

41. Rose EA, Gelijns AC, Moskowitz AJ, et al. Long-term use of a Left Ventricular Assist Device for end-stage heart failure. N Engl J Med. 2001;345(20):1435-43.

42. Rogers JG, Butler J, Lansman SL, et al. Chronic mechanical circulatory support for inotrope-dependent heart failure patients who are not transplant candidates: results of the INTrEPID trial. J Am Coll Cardiol. 2007;50(8):741-7. 